\title{
AGROECOLOGIA: A POLÍTICA PÚBLICA DE ATER LEGITIMANDO O DESENVOLVIMENTO SUSTENTÁVEL NO CAMPO
}

\author{
Silvia Cristina Vieira ${ }^{1}$ \\ Cristiane Hengler Côrrea Bernardo ${ }^{2}$
}

Letícia Flore Junqueira $^{3}$

\begin{abstract}
RESUMO
A extensão rural nacional que em seu inicio monopolizava ações em prol da produtividade, utilizando métodos difusionistas, atualmente trilha uma abordagem diferenciada com inclusão de manejo agroecológico, legitimando um desenvolvimento sustentável no campo, adotando um olhar multidisciplinar que corrobora nas questões ambientais, sociais e econômicas. Este trabalho parte da investigação entre a relação da ATER e da agroecologia e aborda para uma questão reflexiva - As Políticas Públicas contemporâneas de ATER possuem princípios para fomentar a agroecologia em busca de um desenvolvimento sustentável no campo? Objetivouse levantar informações que revelassem a troca de saberes entre a tríade: Políticas Públicas de ATER, agroecologia e desenvolvimento rural sustentável. Como objetivos específicos buscou-se identificar a partir de quando a agroecologia surge com maior força nas ações de ATER nacional. Verificar quais são estas políticas públicas de ATER que pretendem difundir a agroecologia em território nacional. Apontar qual o público alvo atendido por estas políticas públicas. E ainda revelar as legislações que norteiam tais políticas. Por meio de levantamento bibliográfico e documental, com ênfase na legislação de ATER, o presente trabalho refletiu sobre as transformações ocorridas no espaço rural e analisou o estreito vínculo das políticas públicas de ATER contemporâneas, a partir de 2004, com a implantação da Pnater, legitimando o fomento ao desenvolvimento rural sustentável a partir desta práxis em defesa do ideário agroecológico.
\end{abstract}

PALAVRAS-CHAVE: Política Pública Rural. Extensão Rural. Sustentabilidade.

\footnotetext{
${ }^{1}$ Mestranda em agronegócio e desenvolvimento, especialista em gestão do agronegócio e HIPOA/Vigilância sanitária de alimentos, graduada em medicina veterinária. Membro do Projeto de Extensão CoDAF e dos Grupos de Pesquisa CEPEAGRO e PGEA. UNESP. Email.tinavieiragomes@hotmail.com.br

2 Doutora em educação, mestre em comunicação midiática e graduada em jornalismo. Professora assistente doutora. UNESP. Membro dos Grupos de Pesquisa CEPEAGRO e PGEA. E-mail.cristiane@tupa.unesp.br
}

3 Estudante de Administração de Empresas na Universidade Estadual Paulista. UNESP. Email: leticia_flore@hotmail.com 


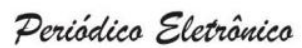

\section{AGROECOLOGY: A PUBLIC POLICY OF ATER LEGITIMIZING THE SUSTAINABLE DEVELOPMENT IN THE FIELD}

\begin{abstract}
The national rural extension that in its beginning monopolized actions in favor of the productivity, using diffusionists methods, currently track a differentiated approach with inclusion of agro-ecological management, legitimizing the sustainable development in the field, adopting a multidisciplinary look that supports the environmental, social and economical issues. This research starts with the investigation of the relationship between ATER and agroecology and it addresses to a reflective question - Do the Contemporary Public Policies ATER have principles to promote the agro-ecology in pursuit of a sustainable development in the field? The objective was to gather information that would reveal the exchange of knowledge between the triad: Public Policies ATER / Agroecology / Sustainable rural development. The specific objectives sought to identify since when the agroecology comes with greater force in the actions of national ATER. Identify what these ATER public policies that intend to spread agroecology in the country. Point the target audience served by these public policies. And yet reveal the laws that guide such policies. Through bibliographic and documentary research, focusing on ATER legislation, this work reflected on the changes occurring in rural areas and analyzed the close relationship of public policies of contemporary ATER since 2004, with the implementation of PNATER, legitimizing the promotion of sustainable rural development from this practice in defense of agro-ecological ideas.
\end{abstract}

KEY-WORDS: Rural Public Policies. Rural Extension. Sustainability.

\section{AGROECOLOGÍA: UNA POLÍTICA PÚBLICA DE ATER LEGITIMACIÓN DE DESARROLLO SOSTENIBLE EN EL CAMPO}

\section{RESUMEN}

La extensión rural nacional que en su comienzo monopolizó las acciones para la productividad, utilizando métodos difusionistas, actualmente el seguimiento de un enfoque diferenciado con inclusión de una gestión agroecológica, legitimando el desarrollo sostenible en el campo, mediante la adopción de una mirada multidisciplinar que apoya las cuestiones ambientales, sociales y económicos. Este trabajo se inicia con la investigación de la relación entre ATER y la agroecología y las direcciones a una pregunta de reflexión - Las Políticas Públicas Contemporáneo ATER tienen principios para promover la agroecología en la búsqueda de un desarrollo sostenible en el campo? El objetivo era reunir información que revelaría el intercambio de conocimientos entre la tríada: Políticas Públicas ATER / Agroecología / desarrollo rural sostenible. Los objetivos específicos intentaron identificar ya que cuando la agroecología viene con mayor fuerza en las acciones de ATER nacional. Identificar cuáles políticas públicas ATER que quieren difundir la agroecología en el país. Punto en el público objetivo servido por estas políticas públicas. Y sin embargo, revelar las leyes que guían estas políticas. A través de la investigación bibliográfica y documental, se centra en la legislación ATER, este trabajo se refleja en los cambios que se producen en las zonas rurales y se analiza la estrecha relación de las políticas públicas de ATER contemporánea desde 2004, con la implementación de PNATER, legitimando la promoción del desarrollo rural sostenible de esta práctica en la defensa de las ideas agroecológicas.

PALABRAS-CLAVE: Política Pública Rural. Extensión rural. Sustentabilidad. 


\section{INTRODUÇÃO}

A política pública de ATER nacional demonstrou, ao longo dos anos, estreito vínculo com o desenvolvimento sustentável na zona rural brasileira, mas somente a partir da elaboração da Política Nacional de Assistência Técnica e Extensão Rural (Pnater) fomentou-se, de maneira coordenada, a agroecologia no campo, legitimando o desenvolvimento rural sustentável no Brasil, por meio de suas diretrizes construtivas.

Neste sentido, a Pnater se articula em torno do ideário do desenvolvimento rural sustentável, da agroecologia, da ação extensionista como um processo educativo e construtivista (ZARNOTT et al. 2015).

Gliessman (2000, p. 55), contribui ao dizer que a agroecologia deriva da relação ecologia-agronomia que tem início na década de 1920. O termo agroecologia foi proposto na década de 1930 como uma "ecologia aplicada à agricultura".

Esta pesquisa parte da investigação entre a simbiose da ATER e da agroecologia e aborda para uma questão reflexiva: Quais os princípios das políticas públicas contemporâneas de ATER podem fomentar a agroecologia em busca de um desenvolvimento sustentável no campo?

E aponta como objetivos específicos:

- A partir de quando a agroecologia surge com maior força nas ações de ATER nacional?

- Quais são estas políticas públicas de ATER que pretendem difundir a agroecologia em território nacional?

- Qual o público alvo atendido por estas políticas públicas?

- Quais as legislações norteiam estas políticas públicas?

O presente artigo buscou discutir as transformações ocorridas no espaço rural e analisar o estreito vínculo das políticas públicas de ATER contemporâneas, nesse ambiente, com o fomento do desenvolvimento rural sustentável a partir dessa práxis em defesa do ideário agroecológico.

Adotou-se como aporte teórico-metodológico, respaldado em levantamento bibliográfico e documental, com ênfase na legislação de ATER, 
utilizando métodos qualitativos que objetivaram, de maneira geral, levantar informações que revelassem a interação entre a tríade: Políticas Públicas de ATER, agroecologia e desenvolvimento rural sustentável.

\section{RELAÇÃo ENTRE A tRÍADE: ATER, AGROECOLOGIA E DESENVOLVIMENTO RURAL SUSTENTÁVEL}

A extensão rural no Brasil, que a priori focalizava ações com viés essencialmente produtivista, utilizando métodos difusionistas ${ }^{4}$ e, prescrevendo insumos químicos sintéticos sem qualquer moderação e respeito ao homem do campo e o meio ambiente, atualmente trilha uma abordagem diferenciada com inclusão de manejo agroecológico, legitimando um desenvolvimento sustentável no campo, adotando um olhar multidisciplinar que corrobora nas questões ambientais, sociais e econômicas.

$\mathrm{Na}$ concepção de Olinger (2001), extensão rural define-se como um processo pedagógico que busca o desenvolvimento rural, utilizando métodos simples que levam o homem do campo a decodificar a mensagem e responder com transformações por meio de amplo processo de participação na identificação de suas necessidades e resolução de suas dificuldades.

Mesmo considerando os relatos de Oliveira (1999), Leme (2005) e Peixoto (2008), que revelam a existência de sinais de extensão rural no Brasil, desde o período imperial e que as atribuições de ações desta prática remontam ao século XIX, este artigo elege o ano de 1930 como marco oficial deste processo.

Oficialmente, no Brasil, pode-se inferir que a extensão rural, de forma institucionalizada chegou por volta do ano de 1930, com a Semana do Fazendeiro, instituída pela Escola Superior de Agricultura de Viçosa, hoje Universidade Federal de Viçosa, em Minas Gerais (SCHAIDHAUER, 2011).

\footnotetext{
${ }^{4}$ Difusionismo é uma forma de comunicação com fluxo unilateral de informações, repassadas de maneira hierárquica, ancorada na imitação. Foi amplamente contestado por Freire (2010) por não apresentar fomento à reflexão no processo pedagógico de ensino - aprendizagem.
} 
Porém, foi em 1948, época que Brasil firmou convênio com os norte americanos que, em Santa Rita de Passo Quatro, interior de São Paulo, ocorreu à primeira experiência extensionista. "O serviço abrangia assuntos de agropecuária e economia doméstica e tinha por objetivo aumentar a produção, a produtividade e a renda das famílias rurais, garantindo-Ihes um melhor nível de vida" (OLINGER, 1996, p.47).

Deste período em diante, muitas conquistas e declínios foram observadas na ATER nacional, até o marco cronológico histórico - quando ocorreu a inclusão da agroecologia como uma ação prioritária da ATER. Até os dias atuais, busca-se nas políticas públicas contemporâneas de extensão rural, incluindo a Pnater, o Programa Nacional de Assistência Técnica e Extensão Rural (Pronater) e a Agência Nacional de Assistência Técnica e Extensão Rural (Anater), uma interface mais sustentável na produção do agronegócio nacional (BRASIL, 2004; 2010; 2014).

Diante destas conquistas, a nova ATER impulsionou um movimento rural mais consciente, por meio da transição e do fortalecimento de uma agricultura convencional, para uma agricultura com manejo agroecológico, legitimando um desenvolvimento rural mais sustentável, delineado pela política pública de ATER, após a implementação da Pnater.

É neste contexto que o Estado brasileiro institui a Pnater, elaborada a partir de um processo participativo e democrático de consultas e debates entre órgãos governamentais, movimentos sociais e empreendimentos de assistência técnica e extensão rural, que permitiu a formulação de novos princípios e diretrizes para a construção de um extensionismo mais comprometido com a busca pela sustentabilidade ambiental (RAMBO et al. 2015).

A Pnater incorpora um conjunto de elementos discutidos no período denominado "Repensar da extensão rural", como o foco no desenvolvimento sustentável, na agroecologia, na valorização dos saberes e das culturas das comunidades tradicionais, nas metodologias participativas, entre outras. No entanto, não recoloca em debate a retomada de um serviço de ATER 


\title{
Periódica Eletrânica

centralizado pelo Estado, ao contrário, reconhece a pluralidade institucional no fornecimento dos serviços de ATER (ZANOTT et al. 2015).

Nesta perspectiva, a relação existente entre a agroecologia e a Pnater é analisada por Coelho e Silveira (2009, p.1), que apontam para o fato de que:

\begin{abstract}
A inserção da noção de Agroecologia no âmbito das Políticas Públicas e em especial na Política Nacional de Assistência Técnica e Extensão Rural propicia um interessante cenário. Passível de assumir distintos contornos, a legitimação da Agroecologia no meio rural se faz de maneira imbricada à disputa entre distintos modelos de agricultura e de desenvolvimento rural.
\end{abstract}

Interessante à análise verificar que, após a introdução da agroecologia nos princípios da extensão rural nacional, tal fato impulsionou e respaldou 0 vínculo entre a ATER e o desenvolvimento da zona rural, harmonizando a produção agrícola e a agropecuária com a qualidade de vida da população do campo, com ênfase na sustentabilidade em seus três níveis: social, ambiental e econômico.

Nota-se forte relevância da Pnater na legitimação do desenvolvimento rural sustentável, nos relatos de Rambo et al. (2015), que salientam que o Estado objetivou oferecer um instrumento de política pública novo e compatível com os ideais do desenvolvimento sustentável. A Pnater estabelece e se baseia em cinco princípios fundamentais que visam orientar um novo compromisso dos extensionistas para com os agricultores e com os resultados socioeconômicos e socioambientais relacionados e derivados de sua ação. Tais princípios são, resumidamente, indicados a seguir:

1. Assegurar, com exclusividade aos agricultores marginalizados pela modernização conservadora, o acesso à assistência técnica e extensão rural pública e gratuita, visando o fortalecimento da agricultura familiar; 2. Contribuir para a promoção do desenvolvimento rural sustentável, com ênfase nos processos de desenvolvimento endógeno; 3 . Adotar uma abordagem multi e interdisciplinar, através de metodologias participativas e de um paradigma tecnológico baseado nos princípios da agroecologia; 4. Estabelecer uma gestão democrática das decisões, contribuir para a construção da cidadania e facilitar o processo de controle social no planejamento, monitoramento e avaliação das atividades extensionistas; 5 . Desenvolver processos educativos permanentes e continuados, a partir de um enfoque dialético, humanista e 
construtivista, visando melhorar a qualidade de vida e promover 0 desenvolvimento rural sustentável (RAMBO et al. 2015, p. 7).

Nesta direção, em busca de um desenvolvimento sustentável, pautado na agroecologia, assim como nos princípios da Pnater, já citados anteriormente, detecta-se nas diretrizes do Pronater, também forte vínculo relacional como:

[...] atuar no processo produtivo e organizacional, com base nos princípios da agroecologia, com o objetivo de orientar o desenho e manejo de agroecossistemas e ecossistemas aquáticos sustentáveis, baseados na abordagem sistêmica e em processos participativos, promovendo o protagonismo dos beneficiários e dos consumidores e construindo, de forma gradativa e de acordo com o desejo dos agricultores envolvidos, estilos de agricultura de base ecológica e sustentável, produzindo alimentos sadios e de qualidade biológica superior (BRASIL, 2005, p. 3).

Percebe-se que ações agroecológicas passam a delimitar os trabalhos da ATER nacional, adotando a nova ciência como ferramenta para a preservação da vida. Tal fato se intensifica após 2004. 
Tabela 1: Políticas Públicas de ATER contemporâneas após 2004

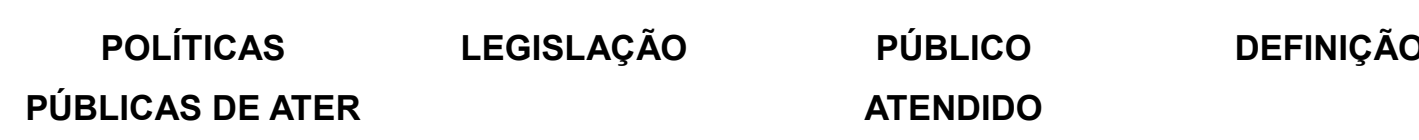

\begin{tabular}{|c|c|c|c|}
\hline PNATER & $\begin{array}{c}\text { Decreto } N^{0} 5.033 \text {, de } \\
5 \text { de abril de } 2004\end{array}$ & Agricultura familiar & $\begin{array}{c}\text { Política Nacional de } \\
\text { Assistência Técnica e } \\
\text { Extensão Rural }\end{array}$ \\
\hline
\end{tabular}

\begin{tabular}{|c|c|c|c|}
\hline \multirow{3}{*}{ PRONATER } & $\begin{array}{l}\text { Lei } n^{\circ} 12.188 \text { de } \\
\text { janeiro de } 2010\end{array}$ & $\begin{array}{c}\text { Agricultores } \\
\text { familiares que se } \\
\text { enquadram na Lei } n^{\circ}\end{array}$ & $\begin{array}{l}\text { Programa Nacional } \\
\text { de Assistência } \\
\text { Técnica e Extensão }\end{array}$ \\
\hline & $\begin{array}{l}\text { Decreto } n^{\circ} 7.215 \text { de } \\
15 \text { de junho de } 2010\end{array}$ & $\begin{array}{c}11.326 \text { de } 24 \text { de } \\
\text { junho de } 2006\end{array}$ & $\begin{array}{c}\text { Rural para a } \\
\text { Agricultura Familiar e } \\
\text { Reforma Agrária }\end{array}$ \\
\hline & $\begin{array}{c}\text { Altera a Lei } n^{\circ} 8.666 \text {, } \\
\text { de } 21 \text { de junho de } \\
1993\end{array}$ & $\begin{array}{l}\text { Ênfase para ATER } \\
\text { Setorial: indígenas, } \\
\text { quilombolas, } \\
\text { ribeirinhos, } \\
\text { aquicultores } \\
\text { extrativistas, } \\
\text { pescadores } \\
\text { artesanais, jovens e } \\
\text { mulheres } \\
\text { trabalhadoras rurais. }\end{array}$ & \\
\hline \multirow{3}{*}{ ANATER } & $\begin{array}{l}\text { Lei } n^{\circ} 12.897 \text { de } 18 \\
\text { de dezembro de } 2013\end{array}$ & $\begin{array}{l}\text { Pequeno e médio } \\
\text { produtor rural }\end{array}$ & $\begin{array}{c}\text { Agência Nacional de } \\
\text { Assistência Técnica e } \\
\text { Extensão Rural }\end{array}$ \\
\hline & Decreto $n^{\circ} 8.252$ de & & \\
\hline & 26 de maio de 2014 & & \\
\hline
\end{tabular}

Fonte: Elaborado pelas autoras, 2015 com dados de (BRASIL, 2004; 2005; 2010; 2014). 
Portanto, estas políticas apresentadas na tabela 1, articulam-se com a ciência agroecológica, pois constituem um elo relacional entre a ATER e a produção sustentável.

Segundo Silva (2014, p. 164), a ciência da agroecologia emerge de uma visão de que nada é superior à vida. Nesta perspectiva o sistema agroecológico vai muito além do despendimento sustentável da zona rural, surge como uma trama de relações, significados e práticas entre todas as formas e modos de vida humana e não humana. A agroecologia admite que a sustentabilidade consiste em cultivar essas relações, significados e práticas que geram a vida, sustentam a vida e dão sentido à existência. "Quando violamos essa trama de vida, criamos vulnerabilidade, e quando dela cuidamos, contribuímos à sua sustentabilidade. Trata-se de uma ciência com consciência".

Como afirmam Sevilla Guzmán e Ottmann (2004), os principais elementos da agroecologia podem ser agrupados em três dimensões:

a) ecológica e técnico-agronômica;

b) socioeconômica e cultural;

c) sócio-política.

Os autores concluem que estas dimensões não são isoladas. $\mathrm{Na}$ realidade elas se mesclam, influenciando uma à outra, de modo que estudálas, entendê-las e propor alternativas que supõe, necessariamente, uma abordagem inter, multi e transdisciplinar, razão pela qual os agroecologistas, os estudiosos da área e seus pares lançam mão de ensinamentos da Física, da Economia Ecológica e Ecologia Política, da Agronomia, da Ecologia, da Educação e Comunicação, da História, da Antropologia e da Sociologia, para ficarmos em alguns dos aportes dos diferentes campos de conhecimento.

De qualquer forma, a agroecologia "não se propõe como uma panacéia para resolver todos os problemas gerados pelas ações antrópicas de nossos modelos de produção e de consumo, nem espera ser a solução para as mazelas causadas pelas estruturas econômicas globalizadas e oligopolizadas", senão que busca, simplesmente, oferecer orientações estratégias de desenvolvimento rural mais sustentável e de transição para estilos de manejos 
agrícolas mais sustentáveis, como uma contribuição para a vida das atuais e das futuras gerações neste planeta de recursos limitados (CAPORAL, 2009, p. 13)

Almeida (2004) aponta que a agroecologia substituiu a noção-chave de tecnologia alternativa, incorporando ideias de educação ambiental (ecológicas, preservacionistas e conservacionistas do meio ambiente) e sociais acerca da agricultura; sua finalidade ultrapassa os limites do viés agrícola.

Neste sentido, a construção do conhecimento agroecológico tende a dialogar com outros saberes e, pelo modo de atuação interdisciplinar da ATER, permeando a utilização de métodos participativos, a agroecologia busca ser compreendida pelos produtores rurais como ciência, minimizando preconceitos sobre as agriculturas alternativas, que racionalizam ou anulam o uso de insumos químicos sintéticos, utilizam o recurso hídrico e os demais recursos naturais de maneira racional e consciente, levando o agricultor à reflexão neste processo pedagógico de extensão rural, adotando um padrão tecnológico que não agrida o meio ambiente $e$, que seja culturalmente adaptada $e$ economicamente viável.

Afirma Gliessman (2000) que a partir dessa concepção, a produção agroecológica é percebida como um processo de aplicação (práticas) de conceitos e princípios ecológicos no manejo de agroecossistemas, no intuito de gerar menos impactos antrópicos negativos no ambiente e na sociedade, e de diminuir o uso de insumos externos a cada estabelecimento rural.

Tais práticas estreitam vínculos e tendem a legitimar o desenvolvimento rural sustentável. Neste sentido, complementa Kageyama (2008) em análise conceitual, que o desenvolvimento rural, não deverá ser pautado somente por meio do desenvolvimento econômico, mas visualizado por meio de um olhar abrangente, permeando um processo que envolve múltiplas dimensões: a econômica; a sociocultural; a politico-institucional e principalmente a ambiental. 


\section{METODOLOGIA}

Para o desenvolvimento da pesquisa, a análise empreendida utilizou o aporte teórico-metodológico respaldada em levantamento bibliográfico e documental com ênfase na legislação de ATER.

Possui objetivo descritivo e exploratório, considerando que a pesquisa exploratória, objetiva tornar familiar o fenômeno ou obter nova percepção do mesmo, desvendar novas ideias. Descritiva por concretizar narrativas das situações e buscar descobrir as relações existentes entre os elementos que compõe a mesma. (CERVO; BERVIAN, 2003).

Adotou-se neste trabalho, uma abordagem qualitativa, pois segundo Marconi; Lakatos (2004) por meio deste método o investigador entra em contato com o ambiente e a situação que está sendo investigada.

$\mathrm{O}$ ambiente rural, ainda propicia descobertas interessantes, pois existem lacunas e há muito a se pesquisar no cenário de sustentabilidade no agronegócio nacional.

\section{RESULTADOS}

Percebe-se que a implementação da agroecologia no cotidiano dos produtores rurais, por meio de ações das politicas públicas de ATER teve inicio em $2004 \mathrm{com}$ a Pnater. No período anterior a esta data, a preocupação maior recaia sobre a produtividade e desprezavam-se parâmetros de sustentabilidade como a introdução à agroecologia.

Destaca-se que o manejo agroecológico vai muito além de uma produção de alimentos mais saudáveis e oriundos de uma "produção limpa", parte-se do princípio que a qualidade de vida do homem do campo, a manutenção de um ecossistema rural harmônico e um custo de produção viável constituem o princípio da sustentabilidade incorporada a um desenvolvimento da zona rural.

Como apontado por Silva (2014), a agroecologia pode contribuir para a construção da qualidade de vida rural, uma alternativa ao desenvolvimento 


\section{Periódica Eletrânica

sustentável, no qual a vida é a origem, centro e fim de todo o pensar e agir do ser humano.

Constatou-se que segundo relato de Caporal (2009) realmente a agroecologia não é a solução para todos os efeitos antrópicos, mas com certeza, minimiza-os e contribui para o fomento de um desenvolvimento rural sustentável.

Porém, muitos produtores ainda carecem de informações e subsídios técnicos a respeito desta modalidade de manejo diferenciada e ecologicamente correta. Fato este que pode ser resultado da falta de reflexões holísticas e da escassez de diálogos entre os agentes envolvidos. E ainda, o público alvo atendido pela ATER é restrito, não abarcando todos os produtores interessados.

As políticas públicas de ATER, a partir de 2004, realizam esta ação de conscientização do produtor rural em prol da adaptação de um manejo produtivo convencional para um manejo agroecológico, construindo uma nova orientação e dinamismo ao serviço oficial de ATER.

Foram elencadas neste artigo as políticas públicas contemporâneas de ATER, que possuem elo relacional com a agroecologia, compondo: a Pnater, o Pronater e a Anater, como descritos na tabela 1, onde se visualiza, além da legislação pertinente a cada política, o público alvo a ser atendido.

Por meio das ações de ATER, das políticas públicas contemporâneas, relatadas na tabela 1 desenha-se uma realidade mais saudável no campo, em busca da conscientização de um manejo mais racional dos recursos naturais, partindo de uma agricultura que priorizava a aplicação de insumos químicos sintéticos para um ambiente mais orgânico e sustentável, estabelecendo simbiose direta entre a tríade: Políticas Públicas de ATER, agroecologia e desenvolvimento rural sustentável.

O atendido das políticas públicas contemporâneas de ATER possuem maior permeabilidade e distinção da heterogeneidade dos produtores, com a chegada da Anater em 2014 que abrange além dos pequenos, assistência para os médios produtores. Embora a Anater ainda não tenha iniciado suas ações a campo, existe uma preocupação ambiental incorporada aos princípios de ATER 
e suas diretrizes de atuação sustentáveis estão sendo desenvolvidas por meio da Empresa Brasileira de Pesquisa Agropecuária - Embrapa.

\section{CONCLUSÃO}

As políticas públicas de ATER, enfatizando a Pnater e as políticas sequenciais a partir do ano de 2004, como o Pronater e a recente Anater constituem intenso vínculo com a agroecologia e consequentemente com o desenvolvimento rural sustentável, pano de fundo deste trabalho.

A agroecologia apresenta-se como uma ciência capaz de unir os diferentes campos de conhecimentos científicos aliados com os saberes tradicionais, na busca pela legitimação do desenvolvimento rural sustentável, apoiados na interdisciplinaridade.

A preconização do uso racional dos recursos naturais e a visão holística, incluindo o produtor rural como parte do ecossistema, delimita uma nova concepção da ATER nacional que por meio do desenvolvimento rural sustentável pretende direcionar esforços para a construção da equidade socioeconômica, visando à superação da discriminação desta categoria social, em defesa do ideário agroecológico, ambientado no espaço geográfico do cenário produtivo do agronegócio.

\section{REFERÊNCIAS BIBLIOGRÁFICAS}

ALMEIDA, J. Agroecologia: nova ciência, alternativa técnico-produtiva ou movimento social? In: RUSCHEINSKY, A. (Org) Sustentabilidade: uma paixão em movimento. Porto Alegre: Sulina, 2004. p. 88-101. Disponível em < http://www.scielo.br/scielo.php?script=sci_nlinks\&ref=000141\&pid=S0034$7590200800030000300002 \&$ Ing=en>. Acesso em jul. de 2015.

BRASIL, PNATER - Política Nacional de Assistência Técnica e Extensão Rural. Ministério do Desenvolvimento Agrário. Versão Final. Brasília: MDA, maio de 2004. Disponível em< http://www.ipea.gov.br/participacao/images/pdfs/conferencias/2CNDRSS/2cndrss\%20poltica_n acional.pdf>. Acesso em jul, de 2015.

PRONATER. 2005. Disponível em < www.faser.org.br/anexos/Pronater.doc. >. Acesso

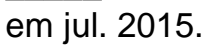

,Lei 12.188 de 11 de janeiro de 2010. Disponível em < http://www.planalto.gov.br/ccivil_03/_Ato2007-2010/2010/Lei/L12188.htm >. Acesso em jul. de 2015. 


\section{Periódica Eletrônica

. Decreto $\mathrm{n}^{\circ}$ 8.252, de 26 de maio de 2014. ANATER. Disponível em <http://www.planalto.gov.br/ccivil_03/_Ato2011-2014/2014/Decreto/D8252.htm>. Acesso em jul. de 2015.

CERVO, A. L.; BERVIAN, P. A. Metodologia científica. São Paulo: Prentice Hall, 2003.

COELHO, V. P.; SILVEIRA, C.A. Agroecologia e a Política Nacional de Assistência Técnica e Extensão Rural, legitimando modelos de agricultura e de desenvolvimento rural? $47^{\circ}$ Congresso SOBER. Porto Alegre. 2009. Disponível em < http://www.sober.org.br/palestra/13/742.pdf>. Acesso em jul. 2015.

CAPORAL, R. C. Agroecologia: uma nova ciência para apoiar a transição a agriculturas mais sustentáveis. In Agroecologia: uma ciência do campo da complexidade. (org.) CAPORAL, R. C. Brasília. DF. 2009. Disponível em

$<$ http://www.emater.tche.br/site/arquivos_pdf/teses/Agroecologiaumacienciadocampodacomple xidade.pdf $>$. Acesso em ago. 2015.

GLIESSMAN, S. Agroecologia: processos ecológicos em agricultura sustentável. Porto Alegre: Ed. UFRGS, 2000.

KAGEYAMA, A. Desenvolvimento Rural: Conceitos e Aplicações ao Caso Brasileiro. Porto Alegre: Editora da UFRGS: Programa de Pós Graduação em Desenvolvimento Rural, 2008.

LEME, J. A. C. O diálogo na ação extensionista como promoção do desenvolvimento rural sustentável no estado de São Paulo. Dissertação Programa de Pós Graduação da Faculdade de Filosofia e Ciências da Universidade Estadual Paulista Campus de Marília, (Área de Concentração: Políticas Públicas e Administração da Educação Brasileira). Marilia: 2005. Disponível em $<$ http://base.repositorio.unesp.br/bitstream/handle/11449/96370/leme_jac_me_mar.pdf?sequen $\mathrm{ce}=1$ \&isAllowed $=\mathrm{y}>$. Acesso em jul. 2015

MARCONI, M. de A.; LAKATOS, E. M. Metodologia Científica. 4ª . ed. São Paulo: Editora Atlas, 2004.

MDA. Ministério do Desenvolvimento Agrário. Programa Nacional de Assistência Técnica e Extensão Rural. PRONATER 2005. Versão 1/3/05.

OLINGER, G. Ascensão e decadência da extensão rural no Brasil. Florianópolis: EPAGRI, 1996.

Métodos de extensão rural. Santa Catarina: EPAGRI, 2001.

OLIVEIRA, M. M. As circunstancias da criação da extensão rural no Brasil. Cadernos de Ciências e Tecnologia, Brasília, v.16, n.02, p. 97 - 134 maio/agosto, 1999. Disponível em < http://seer.sct.embrapa.br/index.php/cct/article/view/8898/5020 >. Acesso em 06 de jan. 2015.

PEIXOTO, M. Extensão rural no Brasil - uma abordagem histórica da legislação. 2008 Disponível em <http://www12.senado.gov.br/publicacoes/estudos-legislativos/tipos-deestudos/textos-para-discussao/td-48-extensao-rural-no-brasil-uma-abordagem-historica-dalegislacao>. Acesso em 05 mai. 2015.

RAMBO, J. R.; DINIZ, R. F.; HESPANHOL, A. N.; SANT'ANA, A. L. Políticas públicas de extensão rural no Brasil contemporâneo: avanços e desafios à construção do desenvolvimento rural sustentável nos estados de Minas Gerais e Mato Grosso. 53ำ Congresso SOBER. João Pessoa. 2015. Disponível em < http://icongresso.itarget.com.br/tra/arquivos/ser.5/1/4598.pdf>. Acesso em ago. 2015. 
SCHAIDHAUER, M. Assistência técnica e extensão no desenvolvimento e promoção do turismo rural. Dissertação de Mestrado. Porto Alegre: UFRGS. 2011. Disponível em < http://www.ufrgs.br/pgdr/dissertacoes_teses/arquivos/mestrado/PGDR_M_138_MAURICIO_SC HAIDHAUER.pdf>. Acesso em jul. de 2015.

SEVILLA GUZMÁN, E.; OTTMANN, G. Las dimensiones de la Agroecología. In: INSTITUTO DE SOCIOLOGÍA Y ESTUDIOS CAMPESINOS. Manual de olivicultura ecológica. Córdoba: Universidad de Córdoba, 2004. p. 11-26. (Proyecto Equal-Adaptagro).

SILVA, J. S. "O dia depois do desenvolvimento: giro filosófico para a construção de uma agricultura familiar agroecológica." Cadernos de Ciência \& Tecnologia 31.2 (2014): 401-420. Disponível em https://seer.sct.embrapa.br/index.php/cct/article/view/20878/12827. Acesso em jul. de 2015.

ZARNOTT, A. V. DALBIANCO, V. P.; NEUMANN, P. S.; FIALHO, M. A. V. Avanços e retrocessos nas políticas de extensão rural: análise crítica sobre a Anater. $53^{\circ}$ Congresso SOBER. João Pessoa. 2015. Disponível em <

http://icongresso.itarget.com.br/tra/arquivos/ser.5/1/5277.pdf>. Acesso em ago. de 2015. 